\title{
Salicin a promising ER, PR and HER 2 binding molecule proving lethal against Hormone + and triple negative breast cancer cells
}

\author{
Research Article
}

\section{Mrudul Pravinbhai Vekaria1*, Pravin Tirgar1}

1. R.K University, School of pharmacy, Bhavnagar Highway, Tramba, Gujarat 360020, India.

\begin{abstract}
Therapeutics against breast cancer is a major research field, due to inefficiency or partial efficiency of existing therapeutics. An urge to discover better therapeutics always persists. Our objective is to study salicin against breast cancer cells, in order to find its therapeutic properties. To study the effect of salicin on breast cancer cells, we performed MTT assay on MCF-7 (hormone positive) and MDA-MB-231 (triple negative) breast cancer cell lines, we did brine shrimp lethality test (BSLT) assay to see the lethal effects of salicin. By the help of bioinformatics we tried to locate the targets that delineate salicin activity. Salicin was docked with estrogen receptor (ER), progesterone receptor (PR) and Human epidermal growth factor receptor 2 (HER2) to study its binding efficiency and possible targets of salicin. Salicin remarkably reduces cell viability both in MCF-7 and MDA-MB-231, along with being lethal to brine shrimps. These results together opine that salicin can be an effective therapeutics against breast cancer cells. The mechanism of action of salicin is probably through ER, PR and HER2 receptors because it can efficiently bind these receptors with minimum energy required for binding. This explains that salicin can easily bind to these receptors. These results together opine that salicin can be an effective therapeutics against breast cancer cells. The mechanism of action of salicin is probably through ER, PR and HER2 receptors because it can efficiently bind these receptors with minimum binding energy. ER, PR and HER2 are major reasons behind the disease pathogenicity depending on the type of breast cancer. According to our results salicin may either induce apoptosis or reduce cellular mitosis both via P53 dependent and independent pathway, which makes salicin a good choice of both hormone positive and negative breast cancer cells.
\end{abstract}

Key Words: Breast cancer, MCF-7, MDA-MB-231, Salicin, In Silico.

\section{Introduction}

Cancer is an evolving threat to humankind and it worsens the socio-economy and psychological health of the suffering patients. Breast cancer is maintaining its position with no reduction in risk of occurrence, recurrence and mortality. According to the breast cancer facts and figures 2019-2020 (American cancer society) (1), an estimated 268,600 new cases of invasive breast cancer will be diagnosed and approximately 41,760 women are expected to die from breast cancer in 2019-2020. According to WHO in 2018 (2), 627,000 women died from breast cancer - that holds $15 \%$ of all cancer deaths among women. Though, breast cancer rates are higher among women in more developed regions. However it is spanning in nearly every region globally from rural to urban. Now the cancer scenario in India is $1,392,179$ for the year 2020 . The common 5 leading sites are breast, lung, mouth, cervix, uteri, and

* Corresponding Author:

\section{Mrudual Pravinbai Vekaria}

R.K University,

School of Pharmacy,

Bhavnagar Highway, Tramba,

Gujarat 360020, India.

Email Id: vekaria mrudul@yahoo.com tongue. Around $57 \%$ of the patients are diagnosed at advanced stage for breast cancer (3).

Several, therapeutics have tried to make their way towards breast cancer treatment. Among them a few are partially successful and most treatments are failures due to resistivity. Breast cancers are treatable only if diagnosed early or at the initial stages. Thus, strategies to treat, manage or prevent the risk of breast cancer are always a need for the global population. In this regard we attempted to find the therapeutic effect of salicin on breast cancer. Salicin is obtained from the bark of Salix species of plants (willow bark). It has been traditionally consumed in China and Europe, throughout centuries for its anti-ache and anti-inflammatory nature. Salicin, also known as salicoside or delta-salicin, is an aryl beta-D-glucoside (salicyl alcohol) in which the phenolic hydrogen is replaced by a beta-D-glucosyl residue. Salicin acts as a prodrug, an antipyretic, a nonnarcotic analgesic, a non-steroidal anti-inflammatory drug (4). However, salicin has been extensively studied as a potent anti-inflammatory agent. The potential medicinal value of salicin as anti-tumorigenic, antiproliferative and antiangiogenic effects still remain unexplored.

Salicin is metabolized to salicylic acid in vivo and then plays a major role, in clinical studies, willow bark extract (source of salicin) has been reported to be effective in pain management and anti-inflammation (5, 
6). Mechanism underlying salicins anti-inflammatory effect was reported in RAW264.7 cells against lipopolysaccharides (LPS) in mouse models where it suppressed the expression of pro-inflammatory cytokines (TNF- $\alpha$, IL-1b, IL-6, and IL-10) and the activation of NF- $\mathrm{KB}$ (7). Administration of salicin suppresses edema and mucosal damage by ameliorating inflammatory response in mouse models of dextran sulfate sodium (DSS)-induced colitis (8). It is quite motivating that willow bark extract has tremendous healing property and other previous studies led us to investigate whether salicin plays a beneficial role in breast cancer and reveal the underlying molecular mechanism of action against breast cancer. Along with anti-inflammation and pain reliving activity, acetylsalicylic acid (ASA, CAS 50-78-2), it has been shown to function same as synthetic non-steroidal antiinflammatory drugs (NSAIDs) and exert antiproliferative effects and to induce pro-apoptosis in a variety of cell lines, e.g., colon, stomach, and prostate cancer cells. These activity of salicin is least explored against breast cancer cell lines or tumors.

About $80 \%$ of all breast cancers are "ERpositive and $65 \%$ are "PR-positive." These cancer cells grow in response to the hormone estrogen or progesterone. Tumors that are ER/PR-positive are much more likely to respond to hormone therapy than tumors that are ER/PR-negative. Medications include Tamoxifen, which blocks hormone receptors by preventing the hormones from binding to them. Aromatase inhibitors such as anastrozole, exemestane, and letrozole are used to treat hormone receptor positive breast cancer in menopausal women to stop estrogen production. Fulvestrant and Toremifene are used to block and damage estrogen receptors and is sometimes used to treat metastatic breast cancer. CDK 4/6 inhibitors such as abemaciclib, palbociclib and ribociclib are sometimes used with aromatase inhibitors or the hormone therapy fulvestrant. Despite of these therapeutic interventions cancer are not curable for $100 \%$ of the population and moreover thee medications carry harmful side effects, for example tamoxifen may induce endometrial cancer. Since, it hampers the normal physiology of both cancer and normal cells (9). Second major protein involved or causes breast cancer is Human epidermal growth factor receptor-2 positive; also known as ERBB2 (HER2) and its relatives HER1 epidermal growth factor receptor (EGFR), HER3 and HER4 are the member of HER family of receptor tyrosine kinases. Activation of this family of receptor tyrosine kinase triggers a rich network of signaling pathways that control normal cells growth including differentiation, motility and adhesion. Alteration of the HER2 oncogene was first studied in breast carcinoma. Involvement of HER2 as a cause of human cell transformation needs further research and investigation. Overexpression of the HER2 oncogene in human breast cancers is associated with a more aggressive form of disease. In approximately $20 \%$ of breast cancers, the cells express a huge amount HER2 which makes them aggressive and fast-growing. In HER2-positive breast cancers, the targeted drug trastuzumab dramatically reduces the risk of the cancer recurrence. Trastuzumab along with chemotherapy is given after surgery to metastatic breast cancer patients. It can also be used for early-stage breast cancer, but it carries a small but real risk of heart damage and possible lung damage. Thus finding an alternative medication with no such side effect is still a global need (10)

The third type of breast cancer is the Triplenegative breast cancer (TNBC) that lack or do not express ER, PR, or HER2 genes. This type of cancers represents challenging clinical condition because they do not respond either to endocrine therapy or other available targeted drugs. They possess metastatic features similar to other breast cancer types but are associated with shorter median time to relapse and death. 10 to $20 \%$ of breast cancer cases are "triple negative and mostly carries BRCA1 mutation. TNBC are often treated with surgery, chemotherapy, and radiation and Olaparib and talazoparip which blocks PARP can be used in BRCA mutation who have metastatic HER2-negative breast cancer. Atezolizumab, an immunotherapy drug in combination with the chemotherapy nab-paclitaxel blocks a protein called PD-L1 are used in certain TNBC. The current medical regimen clarifies that a few drugs with a very few targets are present in the market, and most of them are used in combination with chemotherapy. Chemotherapy being one of the harsh treatment methods generally leaves the patient in a physiologically lost condition. Chemotherapy kills both normal and cancer cells, thus carries severe side effects $(11,12)$. In the current study we used both bioinformatics and in vitro cell culture the access its ER, PR and HER2 binding property and to investigate its effect on hormone receptor positive and negative breast cancer cell line. In the current scenario of adversely affecting drugs or limited drugs, Salicin could be a promising option with multiple targets that it can influence, some of these targets which are directly or indirectly associated with initiation and or progression of the disease.

\section{Materials and Methods}

\section{Chemicals and Reagents}

Salicin (Yucca Enterprises Wadala, Mumbai), MCF-7 cell line (NCCS, Pune), MDA-MB-231 cell line (NCCS, Pune), Amazon.in (e-market) as capsules, RPMI medium with $10 \%$ Fetal bovine serum (FBS) (HiMedia) supplemented with $1 \%$ anti-biotic-antimycotic solution (Sigma-Aldrich), 96 well plate (Thermo Fisher Scientific), Biosafety cabinet level II, MTT solution (HiMedia), Multiskan GO microplate spectrophotometer and Ligplot+ software version 1.4.5 (Roman Laskowski, 2009).

\section{MTT assay}

MTT assay was performed by following a published standardised protocol to check metabolic activity of the cell or cell viability (13). Briefly, An optimum number approximately 5000 cells per well of MCF-7 \& MDA-MB-231 cells in $100 \mu$ media were 
seeded in 96 well plates. Plates were then incubated for $18-24$ hours in $5 \% \mathrm{CO}_{2}$ incubator set at $37{ }^{\circ} \mathrm{C}$. After 24 hours, cells were treated with indicated concentrations of Salicin in triplicate when they reach at about $40-50 \%$ confluence. Plates were again incubated in $5 \% \mathrm{CO}_{2}$ incubator set at $37{ }^{\circ} \mathrm{C}$ for 24 hours. After $24 \mathrm{~h}$ photographs were taken of each treatment group, Thereafter $10 \mu \mathrm{l}$ of MTT $(5 \mathrm{mg} / \mathrm{mL})$ were added in each well in the media. Plate were again incubated for 2-3 hours in $5 \% \mathrm{CO}_{2}$ incubator set at $37^{\circ} \mathrm{C}$ until the formazan crystals are formed that can be seen under the microscope. Media was discarded gently without disturbing formazan crystals. 100-200 $\mu$ of DMSO was added per well to solubilize the crystals. Plates were incubated on a shaker in dark for 10 minutes. Absorbance was noted at $570 \mathrm{~nm}$. $\mathrm{IC}_{50}$ values were then calculated for each sample.

\section{Bioinformatics: In Silico Study: Docking}

Estrogen receptor, progesterone receptor and HER2 protein structures were downloaded from protein data bank website (http://www.rcsb.org/pdb/home/ home.do) in .pdb file format which were directly opened in AutoDock Tool 1.5.6. Structure of Salicin was downloaded from pubchem (website https:// pubchem.ncbi.nlm.nih.gov/ AutoDock Tool 1.5.6 (ATD) was utilized for this study. The AutoDock Vina 1.0 software automatically computed Gasteiger charges, merged non-polar hydrogen's and autodock type to each atom. Then torsions were defined, which showed rotatable and non-rotatable bonds in ligand. Finally, results were saved in pdbqt file format. AutoDock Vina software was run using windows command prompt. All the programme files, ligand (.pdbqt), protein (.pdbqt) and configuration files (.conf) were saved in same folder. The computation was performed in the same folder as log .txt and ligand_out.pdbqt. Log .txt file showed binding energy of ligand to the protein and ligand_out.pdbqt file revealed sites on the proteins with binding energy. The output (.pdbqt) files obtained from the docking study were used to evaluate the hydrophobic interaction of ligand with protein. The results were then processed with chimera software version 1.8 for creation of copies of protein as well as ligand. This was followed by assessment of interactions between protein and ligand by using ligplot + version 1.4.5.

- 3HM1 (human Estrogen Receptor Alpha) with Salicin

- 1A28 (human Progesterone receptor) with Salicin

- 5MY6 (HER2) with Salicin

\section{Brine Shrimp Assay}

Bhrine shrimp assay was performed by following a standardized protocol (14). Brine Shrimp procured from Amazon.in (e-market) as capsules which are filled with thousands of dried cysts. Which were further hatched into nauplii of Brine Shrimp (Artemia salina). Brine Shrimp Lethality Assay (BSLA) has been applied as an alternative bioassay technique to screen the toxicity of drug i.e. Salicin. Dried cysts were performed as indicated above and incubated $(1 \mathrm{~g}$ cyst per litre) in a hatcher at $28-30^{\circ} \mathrm{C}$ with strong aeration, under a continuous light regime. Approximately $12 \mathrm{~h}$ after hatching the phototropic nauplii were collected with a pipette from the lighter side and concentrated in a small vial. Ten brine shrimp were transferred to each well using adequate pipette. In each test 10 Artemia aged $12 \mathrm{~h}$ were exposed in groups to various concentrations of the toxic compound. The toxicity was determined after exposure for $1 \mathrm{~h}, 2 \mathrm{~h}, 5 \mathrm{~h}$ and $8 \mathrm{~h}$ respectively. Percentage of deaths was calculated by counting numbers of survivors in each test group. Death of the Larvae was confirmed if they did not make any internal or external movement during a long observation of several seconds. We ensured that the mortality in the bioassay could be attributed due to bioactive compounds and not because of starvation. A comparison was made between the control and the treated group in terms of dead larvae. The percentage of mortality $(\% \mathrm{M})$ was calculated as:

$\% \mathrm{M}=$ percentage of survival in the control percentage of survival in the treatment.

\section{Alium cepa model}

The antimitotic assay on Alium cepa was perfomrd by following a standardized protocol (15). Briefly, Onion bulbus (Allium cepa L) were obtained from local market and were place in beaker for sprouted in tap water at room temperature. The bulbs were placed on beakers containing the compounds i.e. Salicin (100, $250 \& 500 \mu \mathrm{g} / \mathrm{ml}$ ) such that the roots were slightly immersed in the water containing drug. The duration of treatments for each compound and concentraion was 72 hrs. The sprouted roots were also treated with distilled water and standard drug Methotrexate $(100,250 \& 500$ $\mu \mathrm{g} / \mathrm{ml}$ ), which are used as control and standard respectively. 72.00 hour later the root length and number were counted.

\section{Statistical analyses}

The statistical significance of the data has been determined using one way analysis of variance (ANOVA) followed by post-hoc test using SPSS Software The results are represented as Means \pm S.D.

\section{Results}

\section{MTT assay}

Both MCF-7 cells and MD-MB231 were subjected to MTT assay. In case of MCF-7 cells $100 \%$ of the cells were viable in the control plates (No salicin treatment). Whereas the viability started to reduce with addition of salicin and its increasing concentration having $94.40 \%$ viability in $2.5 \mu \mathrm{M}, 89.28$ in $5 \mu \mathrm{M}$, 88.98 in $10 \mu \mathrm{M}, 87.03$ in $20 \mu \mathrm{M}$ and 62.31 in $40 \mu \mathrm{M}$ of salicin treated plate (Fig.1A). The statistical significance of the data has been determined using one way analysis of variance (ANOVA) followed by Turkey's multiple post-hoc test using SPSS Software. In MTT assay $40 \mu \mathrm{M}$ of Salcin shows clinical significant inhibition of proliferation (Table-1). 


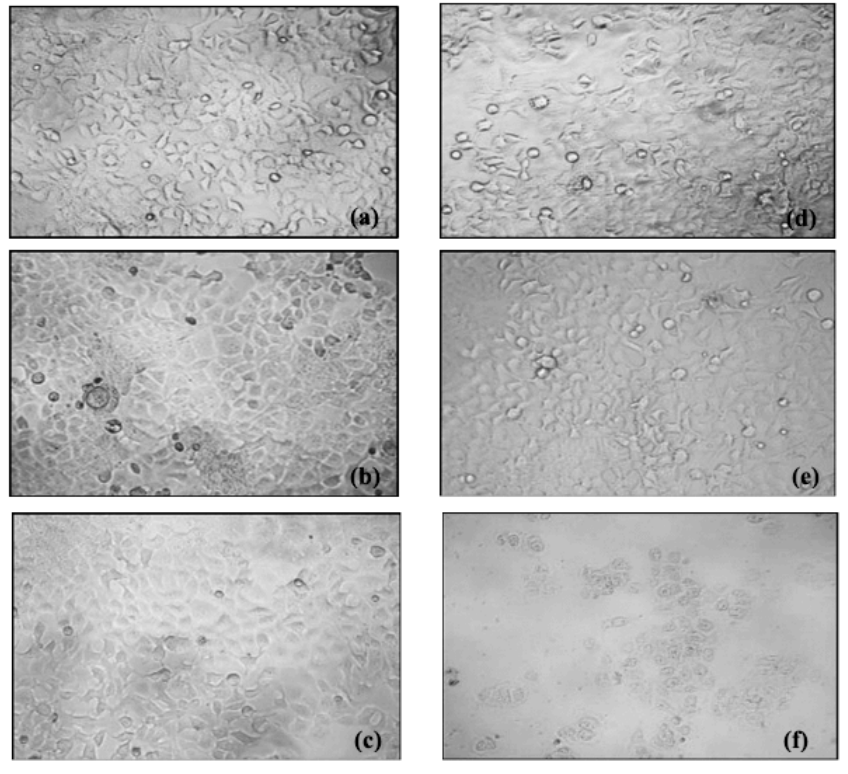

Figure-1A. Effect of Salicin on the presence of viable cells of MCF-7 through formation of formazan crystals after MTT treatment effect of varying concentrations of salicin. (a) control, (b) $2.5 \mu \mathrm{m}$, (c) $5 \mu \mathrm{m}$, (d) $10 \mu \mathrm{m}$, (e) $20 \mu \mathrm{m}$ and (f) $40 \mu \mathrm{m}$.

In case of MD-MB231 the results were similar to MCF7 cell line. $100 \%$ of the cells were viable in the control plates (No salicin treatment). Whereas the viability started to reduce with addition of salicin and its increasing concentration having $92.52 \%$ viability in 2.5 $\mu \mathrm{M}, 87.38$ in $5 \mu \mathrm{M}, 86.70$ in $10 \mu \mathrm{M}, 86.87$ in $20 \mu \mathrm{M}$ and 63.7 in $40 \mu \mathrm{M}$ of salicin treated plate (Fig. 1B). The statistical significance of the data has been determined using one way analysis of variance (ANOVA) followed by Turkey's multiple post-hoc test using SPSS Software. In MTT assay $40 \mu \mathrm{M}$ of salcin shows clinical significant inhibition of proliferation (Table-1).
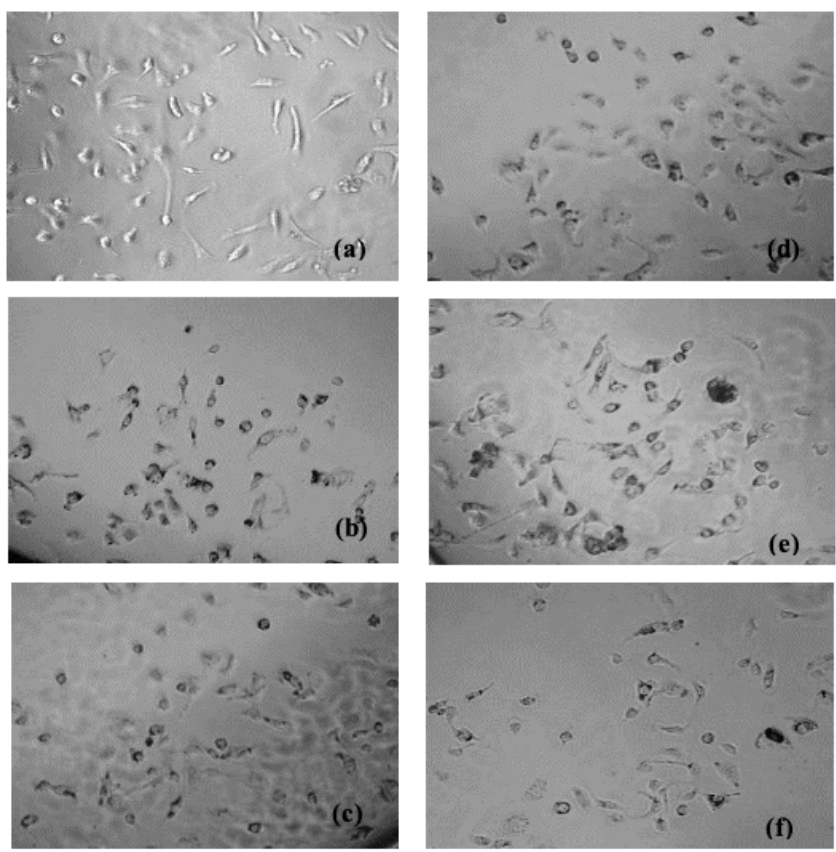

Figure-1B Effect of Salicin on the presence of viable cells of MDA-MB-21 through formation of formazan

crystals after MTT treatment effect of varying concentrations of salicin. (a) control, (b) $2.5 \mu \mathrm{m}$, (c) 5 $\mu \mathrm{m}$, (d) $10 \mu \mathrm{m}$, (e) $20 \mu \mathrm{m}$ and (f) $40 \mu \mathrm{m}$.

Table-1: MTT assay for MCF-7 Cell line

\section{Concentrations}

No drug treatment (Negative control)

$2.5 \mu \mathrm{M}$

$5.0 \mu \mathrm{M}$

$10 \mu \mathrm{M}$

$20 \mu \mathrm{M}$

$40 \mu \mathrm{M}$

IC 50
Average Absorbance

-MCF 7 MDA-MB-231

$0.769 \pm 0.021$

$0.726 \pm 0.098$

$0.686 \pm 0.014$

$0.684 \pm 0.052$

$0.669 \pm 0.000$

$0.479 \pm 0.001^{* *}$

$>40 \mu \mathrm{M}$
Percent Cell Viability (\%)

\begin{tabular}{|l|l|}
\hline \multicolumn{1}{|c|}{-MCF 7 } & \multicolumn{1}{|c}{ MDA-MB-231 } \\
\hline 100 & 100 \\
\hline 94.40 & $92.52 \pm 10.6$ \\
\hline 89.28 & $87.38 \pm 2$ \\
\hline 88.98 & $86.70 \pm 8$ \\
\hline 87.03 & $86.87 \pm 1.5$ \\
\hline 62.31 & $63.17 \pm 0.8$ \\
& $>\mathbf{4 0} \boldsymbol{\mu M}$ \\
\hline
\end{tabular}

Values are expressed as mean \pm SD $(n=3)$. Statistical analysis: One-way ANOVA followed by Tukey's post hoc test. $* p<0.05, * * p<0.01, * * * p<0.001$ as compared to control.

\section{In Silico Data: Receptor ligand docking with Autodock and interaction study by Ligpot}

Predictions of binding energies of salicin with different molecules were estimated using AutoDock Vina and results are shown in table-2, along with its interactions with protein residue.

Table-2: Docking of salicin with ER, PR and HER2 receptor

\section{Complex}

Progesterone receptor -

Salicin

Estrogen receptor -

Salicin

HER2 receptor -

Salicin

\section{Energy}

(Kcal/mol) Hydrogen Bonding

$-7.9$

$-7.0$

$-7.3$
Gln725 (2) Asn719

Arg335, Ser341

Thr5 (2), Tyr281, Gly440, Asn466
Interaction bonds

Hydrophobic interaction

Met759, Gly722, Phe778, Leu178,

Met909, Leu721

Phe337, Ser338, Asp332, Pro336,

Met342, Leu345, Try331

Phe269, Tyr279, Ser441, Cys4 
Interaction bonds contain the number of amino acids with hydrogen bonding and hydrophobic bonding. The data has been shown the lowest level of energy in binding site.

Salicin showed hydrophobic interaction and hydrogen bonding with estrogen, progesterone and HER2 receptor. It is very clear that hydrophobic interactions play a major role in stabilizing the ligands at the binding interface. Autodock provides output for 10 binding sites among these 10 binding sites the one was selected based on least binding energy required which can be the site with best chance of ligand binding. Thus, it confirms a stable binding of salicin with these three receptors. These receptors are critical to breast cancer initiation and or progression. A number of hydrophobic atoms are present in substituted compounds and may be important for drug-target binding (Fig-2).

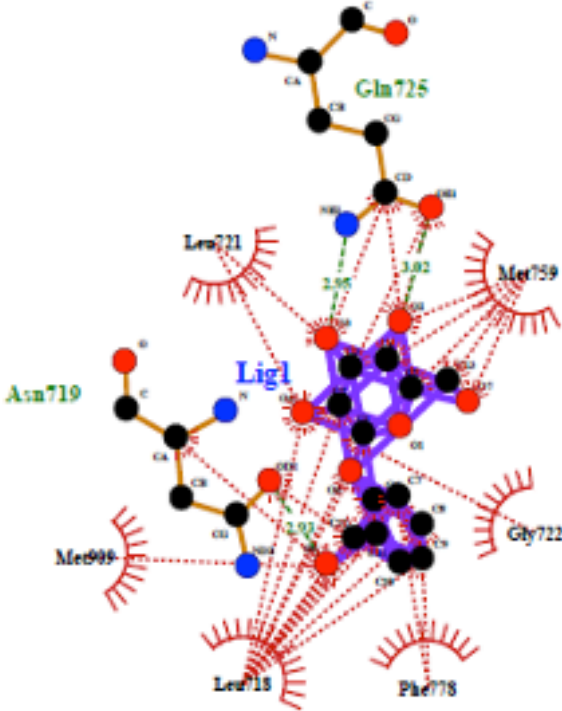

Salicin_Progesterone

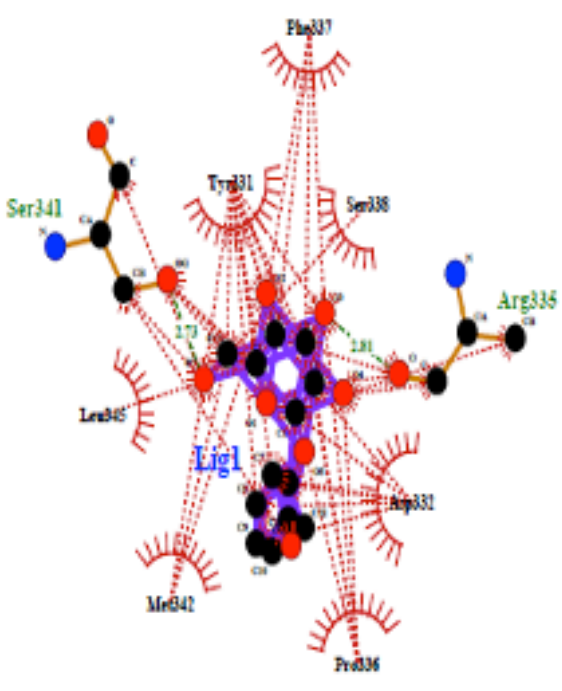

Salicin_Estrogen

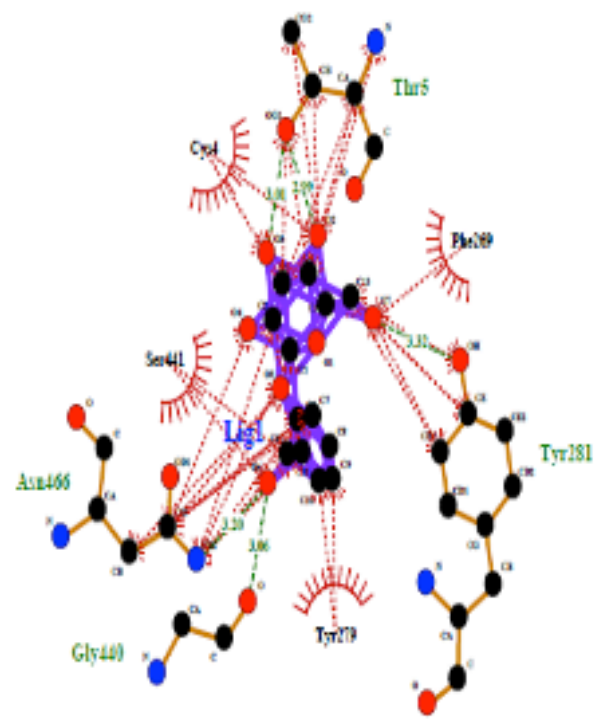

Salicin_Her2

Figure-2: Receptor protein and ligand interaction by Ligpot software output. Hydrogen bond (dotted green line) and hydrophobic bonds (dotted red line) as interaction of salicin with (A) Progesterone (B) estrogen (C) HER2 residues.

\section{Brine shrimp assay (BSLT)}

BSLT is a trusted lethality assay mostly performed before drug trials in research studies. $100 \mu \mathrm{g} / \mathrm{mL}$ of salicin proves to be LC50 at 5 hours of treatment killing $54 \%$ of napulie whereas $500 \mu \mathrm{g} / \mathrm{mL}$ of salicin seems to be the LC50 at 2 hours of treatment killing almost $45 \%$ of napulie at 2 hours and $64 \%$ at 5 hours of treatment (Table- 3 ).

Table-3: \% Mortality Due to Effect of Salicin on Brine shrimp

\begin{tabular}{|c|c|c|c|c|}
\hline \multirow{2}{*}{ Time Point } & \multicolumn{4}{|c|}{ Salicin } \\
\cline { 2 - 5 } & Control & $\mathbf{1 0 0} \boldsymbol{\mu g} / \mathbf{m l}$ & $\mathbf{2 5 0} \boldsymbol{\mu g} / \mathbf{m l}$ & $\mathbf{5 0 0} \boldsymbol{\mu g} / \mathbf{m l}$ \\
\hline $\mathbf{1 . 0 0} \mathbf{~ h r}$ & $0.0 \%$ & $13 \%$ & $13 \%$ & $27 \%$ \\
\hline $\mathbf{2 . 0 0} \mathbf{~ h r}$ & $0.5 \%$ & $28 \%$ & $35 \%$ & $45 \%$ \\
\hline $\mathbf{5 . 0 0} \mathbf{~ h r}$ & $13 \%$ & $54 \%$ & $50 \%$ & $64 \%$ \\
\hline $\mathbf{8 . 0 0} \mathbf{h r}$ & $20 \%$ & $60 \%$ & $57 \%$ & $77 \%$ \\
\hline
\end{tabular}

\section{Alium cepa root model for morphometric antimitotic activity.}

Fresh onions were treated with different concentration of salicin such as 100,250 and $500 \mu \mathrm{g} / \mathrm{ml}$ for 72 hours. The sprouting of the roots was gradually reduced in the treated onions $(25,20$, and 10$)$ as compared to the control (34 roots) (Table-4, Fi3). Root length was also reduced in the treated group with gradual increase in the concentration of salicin in comparison to control. It was $4.80 \pm 0.78$ in control whereas $3.41 \pm 0.77$ in 100, $2.34 \pm 0.5$ in 250 , and $1.94 \pm$ 0.66 in $500 \mu \mathrm{g} / \mathrm{ml}$ of salicin. These results strongly indicate the antimitotic effect of salicin. Antimitotic affect may become beneficial in breast cancer treatment. 
Mrudual Pravinbai Vekaria et.al., Salicin: a promising ER, PR and HER2 binding molecule

Table-4: Effect of Salicin on root length and root number of Allium cepa root

\begin{tabular}{|c|c|c|c|c|}
\hline \multirow{2}{*}{ Groups } & \multicolumn{4}{|c|}{ Concentration } \\
\hline & Control & $100 \mathrm{mcg} / \mathrm{ml}$ & $250 \mathrm{mcg} / \mathrm{ml}$ & $500 \mathrm{mcg} / \mathrm{ml}$ \\
\hline Root number & 34 & 25 & 20 & 10 \\
\hline Root Length (cms) & $4.80 \pm 0.78$ & $3.41 \pm 0.77$ & $2.34 \pm 0.5$ & $1.94 \pm 0.66$ \\
\hline
\end{tabular}

SD is standard deviation for $\mathrm{N}=10$ observations
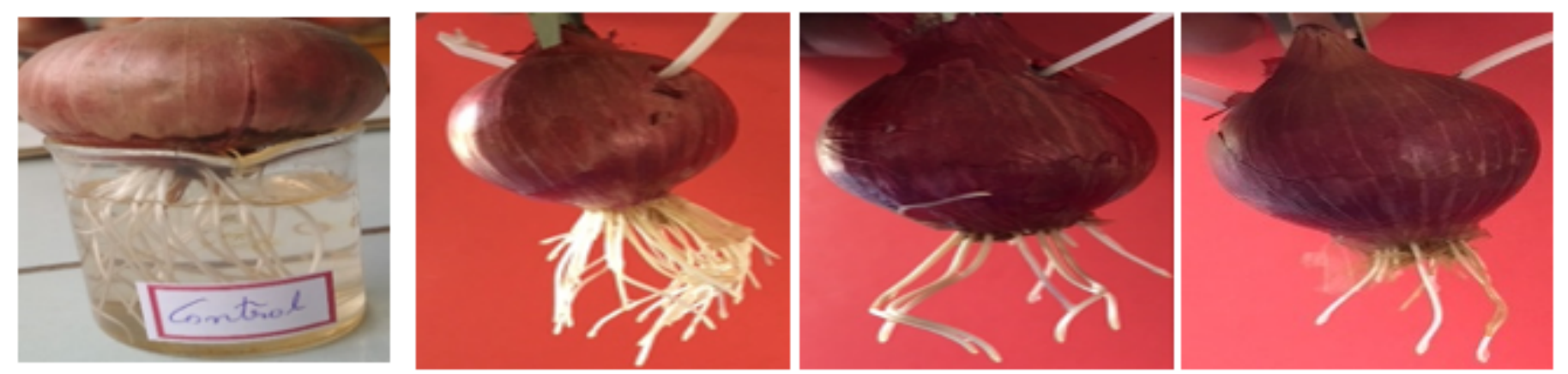

Figure-3: Treatment of Alium cepa root with different concentration of Salicin (a) Control (b) $100 \mu \mathrm{g} / \mathrm{ml}$ (c) 250 $\mu \mathrm{g} / \mathrm{ml}$ (d) $500 \mu \mathrm{g} / \mathrm{ml}$.

\section{Discussion}

The present study aimed to explore the antiproliferative, antimitotic and cytotoxic effect of salicin on hormone receptor positive MCF-7 and triple negative MDA- MB231 breast cancer cell line. MCF-7 cell line is positive for estrogen, progesterone and also for the glucocorticoid receptors. It is a perfect cell lineage to represent hormone responsive breast cancer (16). The MDA-MB-231 cell line is generally used to model late-stage breast cancer and is negative for ER, PR, and HER2. Studying the effect of salicin on MDAMB231 gives us an idea about the ER, PR and HER2 independent pathway of salicin's effect on cell viability. The current study showed markedly reduced MDAMB-231 and MCF-7 cell viability with increasing concentration of salicin. The difference in cell viability between untreated and treated cell line was found to be significant at $40 \mu \mathrm{M}$ concentration. The reduced cell viability can be attributed either by salicin being cytotoxic to cell thereby inducing cellular apoptosis or it indicates towards reduced cell division or mitosis with time lapse. The cellular features also appear to be different from normal with gradual increment of salicin dose, but not clearly visible as blebbing which occurs in apoptosis.

Apoptosis involves modulation of caspases and kinases and is a tightly regulated physiological mechanism (17). In normal condition cell proliferation and apoptosis remains in a balanced state, thus apoptosis is critical in cancer pathogenicity (18). Carcinogenesis begins when this balance shifts in favour of reduced apoptosis and enhanced survival of damaged cells or potential neoplastic cells (19). In an earlier study salicin and fenofibrate were shown to be cytotoxic to MCF-7 and Panc-1 cell lines through activation of the caspase $3 / 7$ apoptotic pathway (20) which supports our current results of reduced cell viability in MCF-7 cells (Fig-1a, 1b). Another study showed that willow bark extract BNO 1455 and its other fractions inhibit the cell growth and promote apoptosis in human colon and lung cancer cell lines irrespective of their COX-selectivity (21). Though this study does not directly claims that salicin does so, but salicin is one of the major components of willow bark, and thus salicin may promote apoptosis despite of cox selectivity.

Salicin exhibits anti-inflammatory action by inhibiting COX-2 and its expression via NF- $\mathrm{KB}(22)$. The activation of NF- $\kappa \mathrm{B}$ evidences the potential connection between inflammation and cancer. Formation of NF- $\kappa B$ is the consequence of an inflammatory microenvironment for malignant transformation. The expression of cancer-promoting cytokines is upregulated by NF- $\kappa \mathrm{B}$. These cytokines include IL- 6 or TNF- $\alpha$ along with the survival genes such as Bcl-XL (23).

An earlier study have compared acetyl salicylic acid (ASA) to diclofenac (Diclo, CAS 15307-79-6), and found that the COX-1 and COX-2 mRNA expression patterns were different when treated with different willow bark extract. ASA and diclofenac both inhibited COX-1 and COX-2 mRNA expressions. Whereas, STW 33-I and its fraction E increased the COX-1 mRNA expression. Different willow bark extract have different influence on the COX-1 and COX-2 mRNA expressions in comparison to NSAIDs, which might be helpful in the prevention of undesirable side effects such as gastric erosions (24). From all the above studies, salicin seems to hold a regulatory effect on cox and $N F-\kappa B$, which are quite important in carcinogenesis. Through controlling the expression of target genes, such as TNFA, IL6, BCLXL, BCL2, BCLXS, XIAP, and VEGF, NF- $\kappa B$ mediates tumor-cell proliferation, survival, and angiogenesis (25).

For developing drug from crude plant extracts, the extracts are first screened for their cytotoxic activity and then further assayed using cancer cell lines or animal models. Brine Shrimp Lethality Test (BSLT) has a good correlation with tumour cell lines of various types. According to BSLT the cytotoxic activity of an 
extract is determined by $50 \%$ brine shrimps mortality response (LC50). If a plant extract had $\mathrm{LC}_{50}<1000 \mu \mathrm{g} /$ $\mathrm{mL}$ it is considered toxic to brine shrimps (26). This toxicity level may show anticancer potentials. In the current study we found that $100 \mu \mathrm{g} / \mathrm{mL}$ of salicin proves to be $\mathrm{LC}_{50}$ at 5 hours of treatment killing $54 \%$ of napulie whereas $500 \mu \mathrm{g} / \mathrm{mL}$ of salicin seems to be the $\mathrm{LC}_{50}$ at 2 hours of treatment killing almost $45 \%$ of napulie at 2 hours and $64 \%$ at 5 hours of treatment (Table-3). However, salicin showing a strong cytotoxic activity seems to be highly anticancerous or have killing property, thus it pops a question that it may be cytotoxic even to the normal cells. Salicin and fenofibrate decreases tumor weight, tumor volume, CEA level, and cholesterol content through antioxidant like activity (reduced MDA level and increased GSH, catalase content) and anti-inflammatory activity (reduced TNF$\propto$ level) which evidences an in vivo anticancer activity (20). The cytotoxic activity paired with ROS induction, have higher chances of being cytotoxic to both normal and cancer cells. However, salicin acting as antioxidants may not have any cytotoxic activity on normal cells or shall have least of it.

Interestingly, ethanolic extract from the bark (EEB) showed the highest anticancer effect on colon cancer cells but not on normal colon fibroblast cell line. Possibly EEB could strongly reduce the proliferation of the cancer cells only. EEB was able to arrest cell cycle at $\mathrm{G} 1 / \mathrm{S}$ independent of DNA damage in the cancer cells, and induced apoptosis through a p53 dependent pathway along with the inhibition of PI3K/Akt and MAP Kinase pathways similar to known commercial inhibitors. This study proposed that, the combination of the polyphenols and flavonoids in EEB contributes toward its potent anticarcinogenic effects (27). Though this study showed that EEB viz also a source of salicin induces apoptosis via p53 dependent pathway but amazingly in our study salicin reduced the cell viability even of the MDA-MB231 cell line also (Table-1) which is ER, PR, HER2, E-cadherin negative and expresses mutated p53. Now a mutated p53 cannot function similar to a normal one. P53 is best known as cell cycle regulator and an initiator of apoptosis and senescence. Strangely in case of MDA-MB231 salicin does not work via p53 mediated pathway (28). To think beyond p53 mediated pathways we found that in our resuts salicin has shown strong antimitotic properties on the onion root tip (Fig- 3). The antimitotic property of salicin may be attributed by its potentials to arrest cell cycle at G1/S (27). Mechanism of delayed mitosis is linked to cell death referred as mitotic catastrophe. Salicin may be involved in Mitotic catastrophe in MDA-MB231.

Cells either with wild-type or mutated p53 when transfected with siRNAs targeting $\gamma$-tubulin, Aurora A, ninein, TACC3, TOG, or pericentriolar material-1, showed that knockdown of these led to cell death, regardless of p53 status. Possibly this may be the pathway of salicin being cytotoxic against MDAMB231. In the current study salicin showed antimititoc property and remarkably reduced the root number as well as root length of onion treated with salicin in comparison to control (Table-4) along with reduced cell viability. The same may or may not be the case in MCF-7 cells, because it has a normal p53 status. Metaphase arrest is necessary for the mitotic catastrophe and cell death caused by depletion of centrosomal proteins leads to elevated phosphorylation of Chk2. Phosphorylated Chk2 localization was also observed to be increased in the nuclei of dying cells and at the centrosome in $\mathrm{M}$ phase arrested cell. Centrosomal protein depletion with cotransfection of siRNAs against Chk2 decreases the amount of cell death (29). Thus, Chk2 activity is essential for cell apoptosis after mitotic catastrophe due to depletion of centrosomal proteins that troubles microtubule organization.

Cell death due to mitotic catastrophe, thus often takes place in conjunction with apoptosis. A study reported that the UV-inactivated parvovirus (adenoassociated virus; AAV) infected Osteosarcoma cells (U2OSp53DD) died through mitotic catastrophe with no indication of DNA fragmentation or chromosome condensation. Furthermore, cell death was free of caspases, apoptosis-inducing factor (AIF), autophagy and necroptosis. Taken together the results indicate that in the absence of the G1 checkpoint viz possible by salicin or willow bark extract, mitotic catastrophe takes place in these p53-null cells (possible in MDAMB-231) chiefly due to mechanical disruption induced by centrosome overduplication, and not as a consequence of a suicide signal (30).

The current bioinformatics result have shown significant estrogen receptor (ER), progesterone receptor and HER2 receptor binding efficacy of salicin (Table-2, Fig-2) and possibly acts either as an antagonist or an agonist of ER,PR and HER2. Thereby inhibiting the ER, PR and HER2 induced signaling pathways or expression of target proteins. ER normally allows E2 binding and exerts profound effects on cell growth, development, differentiation, and homeostasis. E2-ER modulates the activities of transcription factors such as the activator protein (AP)-1, nuclear factor- $\kappa \mathrm{B}$ (NF- $\kappa \mathrm{B})$ and stimulating protein-1 (Sp-1), by stabilizing DNA-protein complexes and/or recruiting co-activators. In addition, E2-ER conjugations leads to activation of several signal transduction pathways (e.g. ERK/MAPK, p38/MAPK, PI3K/AKT, PLC/PKC) (31). E2 is an established intrinsic carcinogen and has vital roles in the E2 dependent ER positive cancers. Moreover BRCA mutation also leads to cancer in E2 dependent or synthesizing organs such as breast and ovary (32). Thus E2 has some strong link with cancers, whether it is hormone positive or not or due to BRCA mutation. Oxidative-stress initiated by E2 is evident from earlier studies representing enhanced lipid-peroxidation and other stress parameters; E2 also exerts ER mediated oxidative stress (33). Estrogen receptor $\alpha(\mathrm{ER} \alpha)$ results in estrogen-mediated transcriptional signalling and over expression of the important drug-metabolizing enzyme, glutathione S-transferase P1-1 (GSTP). This enzyme utilizes GSH for the conjugation reactions in tumors. GSTP over expression has been correlated to the poor prognosis in breast cancer (33). The E2 associated GSH increase has been indicative in this regard. 
Evidence suggests that both ROS and CRP can activate the transcription factor NF- $\kappa-\beta(34)$ and several mitogen activated protein kinases (MAPKs) (34). So the antioxidant activity of salicin additionally prevents the activation of these signaling pathways along with the ER inhibition. (33). Breast cancer pathogenicity is associated to the tumoral estrogen sulfotransferase (hSULT1E1) whose expression has been shown to be regulated by cellular redox status which is interlinked to or dependent on the Nrf-2/NF-kß interplay (34). Salicin seems to activate and increase nuclear translocation of Nrf2, thus has a direct effect on the expression of SULT1E1, which in turn sulfonates and inactivates estrogen. Estrogen is capable of regulating enzymes (SULT1E1, STS) and protein that in turn are capable of regulating estrogen and it holds a promising role in breast cancer initiation, or progression and severity and interestingly all these events are partially controlled by oxidative stress. Salicin may inhibit the estrogen activity in a dual way by being an antioxidant and by its strong ER binding capacity (Fig-3) it can antagonize E2 and inhibit ER function.

An earlier study injected Ehrlich Ascites Carcinoma Cells (EACC) into the intraperitoneal cavity of mice. These mice were then fed with willow extract via stomach tube. EACC induced tumor growth was reduced by willow bark extract and it also delayed death (for 35 days). In vitro the willow extract was able to kill majority (75\%-80\%) of abnormal cells obtained from patients with acute lymphoblastic leukemia (ALL) and with acute myeloid leukemia (AML). DNA fragmentation pattern revealed that cellular apoptosis have occurred (32). Hyper vascularization and remodeling of the vascular system in tumors by increased angiogenesis, enhances it survival capability. Overexpression of VEGF increased tumor growth and angiogenesis significantly in a murine model of breast cancer. 17beta-estradiol (E2) modulates VEGF expression in in MCF-7 cells and in MDA-MB-231 cotransfected with estrogen receptor (ER $\alpha$ or ER $\beta$ ) through transcriptional activation. The positive effect was abolished when MCF-7 cells were treated with the pure antiestrogen ICI 182,780 or the agonist/antagonist tamoxifen (36). This study also identified an estrogen responsive element (ERE1520) present on the VEGF promoter. ERE1520 formed a complex with ER $\alpha$ or ER $\beta$ proteins in gel shift assay using MCF-7 or MDAMB-231 nuclear extracts. Eventually suggesting that in breast cancer cells VEGF is a target gene for ER $\alpha$ or ER $\beta$. Salicin a known natural antipyretic that resembles aspirin (37), suppresses the angiogenic activity of endothelial cells, such as migration, tube formation, and sprouting from an aorta. Moreover, salicin reduced ROS production and activation of the extracellular signalregulated kinase pathway. The expression of VEGF was also decreased by salicin in endothelial cells. Salicin inhibited tumor growth and angiogenesis in a mouse tumor model. Taken together, salicin targets the signaling pathways mediated by reactive oxygen species and extracellular signal-regulated kinase, providing new perspectives into a potent therapeutic agent for hyper vascularized tumors (38). Since VEGF is a target gene for ER, and Salicin has significant binding capacity to ER, it is possible that salicin inhibits angiogenesis by antagonizing ER.

Therapeutics against triple-negative breast cancer is challenging, EGFR inhibition has been proposed as a mechanism. Triple-negative breast cancer is clearly a distinct clinical subtype, from the perspective of both ER and HER2 expression. Ethanol extract from bark (EEB) of Salix aegyptiaca can induce apoptosis and inhibits proliferation in colon cancer cells. Tandem mass spectrometry revealed EEB to be rich in catechin, catechol, and salicin which together amplified neoplastic cell apoptosis in the colon mucosa of DMH-treated mice and lowered the levels of EGFR, nuclear $\beta$-catenin, and COX-2 in HT-29 and HCT-116 cell lines (colon cancer cells) (39). In the current study, the main features that distinguish salicin treated and untreated cell lines are the size, number and the characteristics of their shapes (Fig- 1b, 2b). Such changes in cellular characteristics have also been noticed in earlier studies (17). Salicin significantly reduced cell viability and the of expression cytokines, TNF- $\alpha$, IL-6 and MMP1-3 in IL-1 $\beta$-induced rheumatoid arthritis fibroblast-like synoviocytes (RA-FLSs). It inhibited ROS production, p65 phosphorylation in comparison to IL-1 $\beta$ treatment. Salicin promoted nuclear translocation of $\mathrm{Nrf} 2$ and expression of HO-1 with lowering ROS production in IL- $1 \beta$-induced RAFLSs. Salicin successfully suppressed an in- vivo oxidative damage indexes of ankle joint cells (40).

HER2-driven proliferation, vessel formation and/or invasiveness however may lead to poor prognosis. HER2 over expression is characterized by peculiar gene expression profile. HER2-positive tumors were shown to be resistant to endocrine therapy anthracyclin treatment efficiently worked against it. Despite the fact that several pathways interacting with HER2 are still not completely understood. This tyrosine kinase receptor is, to date, a promising molecule for targeted therapy. In the current study salicin shows efficient binding with HER2receptor thus possibly may directly inhibit HER2 driven pathways, Overexpression of HER2 in 3D models induces proliferative and antiapoptotic changes that mimic early stages of epithelial cell transformation $(41,42)$. HER2 overexpression contributes to several signaling abnormalities due to increased HER2 dimers. These dimers disrupt cellular polarity, HER2-HER3 dimers are drivers of proliferation, survival, invasiveness in cancers. Rare $\triangle$ HER2 isoform possesses more potent signaling characteristics and an increase in it results in a plethora of abnormal gene expression (43) According to the current study salicin is able to bind to HER2 and possibly may inhibit the HER2 function and its consequences. Along with ER, PR and HER2 effective binding, Salicin in vivo experiment blocked NF- $\kappa B$ and MAPK pathways activation. MAPKs include p38 MAPK, ERK, and JNK, and critically regulate inflammatory responses. Phosphorylated MAPK molecules lead to the production of pro-inflammatory mediators and cytokines (7). Salicin having control of $\mathrm{NF}-\kappa \mathrm{B}, \mathrm{NRF} 2$, other inflammatory cytokines, ability to 
arrest cell cycle at G1/S, capable of inducing apoptosis, inhibit h42er vascularization, having binding affinity to vital molecules critical to breast cancer such as ER, PR and HER 2 which mediates proliferatory signaling.

A bunch of evidences link progesterone to breast cancer. Several PR-regulated genes are associated with tumor progression resulting in aggressive tumor phenotype. Variation in PR-A: PR-B ratio is noticed, more in breast tumors in comparison to normal tissue (44), this may modify the genetic program in favor of being cancerous $(45,46)$. Both estradiol and progesterone are vital in the mammary gland for cellular proliferation (47). Analysis of epithelial subpopulations in BRCA1 mutation carriers showed an abnormally elevated luminal progenitor cells (functionally intact) with RANK expression and disrupted progesterone regulation, and elevated DNA damage. This scenario in BRCA1 carriers is due to RANKL expression mediated by progesterone in the terminal ER+ PR+ luminal cell fraction $(48,49)$. Elevated expression of RANKL mRNA and high levels of serum progesterone is highly correlated in normal human breast samples (fine-needle aspirate) (50). RANKL protein or mRNA expression is higher in breast tissue in high progesterone conditions such as during pregnancy, luteal phase of menstrual cycle, and due to combined hormone therapy (51) progesterone has biphasic effects on the in vitro proliferation of breast cancer cell lines i.e., progesterone is both proliferative as well as inhibitory. Whereas, estrogen has mitogenic effects on same cell-line grown in vitro. These studies suggest that progesterone primarily acts as a priming agent, with growth-promoting activity dependent upon cellular background or initially promotes proliferation $(52,53)$. Therefore progesterone has potential roles in the breast cancer initiation and or balancing the cancerous condition, in that case Salicin being able to bind progesterone receptor may lead to inhibition of progesterone's priming effects and or initiation of cancer attributed by its proliferation properties. The binding energy of salicin and PR was -7.9 , that seems to be the least among ER- salicin (-7.0), and HER2-Salicin (-7.3). Together salicin seems to be a promising drug against ER+, HER2+ and triple negative breast cancer.

\section{Conclusion}

There is a critical driving role of ER and HER2 in human cancers, and the large number of patients affected by this type of cancer has made estradiol, ER, PR and HER2 an ideal target for the development of rationally designed anti-cancer drugs. Salicin with an effective binding to ER, PR and HER2 and being lethal to MCF-7 and MDA-MB- 231 Cell line seems to be an upcoming therapeutics against breast cancer. Our results interprets that salicin may either induce apoptosis or reduce cellular mitosis both via P53 dependent and independent pathway, which makes salicin a good choice for both hormone positive and negative breast cancer cells.

\section{Acknowledgments}

The authors thank B. V. Patel PERD Center, Ahmedabad for providing professional support for cell line study.

\section{Declaration of interest}

The authors declare that there is no conflict of interest.

\section{References}

1. American Cancer Society. Breast Cancer Facts \& Figures 2019-2020. Atlanta: American Cancer Society, Inc. 2019.

2. World Health Organization. Global Health Observatory. Geneva: World Health Organization; 2018. (Last accessed on November 28, 2020). Available from: http://who.int/gho/database/en/.

3. Prashant M, Krishnan S, Meesha C, Priyanka D, Kondalli LS, Stephen S, Vinodh N, Anish J, Sandeep N, Francis SR, and on behalf of ICMRNCDIR-NCRP Investigator Group. JCO Global Oncology 2020; 6:1063-75.

4. Akao T, Yoshino T, Kobashi K, Hattori M. Evaluation of salicin as an antipyretic prodrug that does not cause gastric injury. Planta Med. 2002;68(8):714-8.

5. Vlachojannis JE, Cameron M, Chrubasik S. A systematic review on the effectiveness of willow bark for musculoskeletal pain. Phytother Res. 2009; 23(7):897-900.

6. Fiebich BL, Appel K. Anti-inflammatory effects of willow bark extract. Clin Pharmacol Ther. 2003; 74 (1):96-7.

7. Li Y, Wu Q, Deng Y, Lv H, Qiu J, Chi G, Feng H. D (-)-Salicin inhibits the LPS-induced inflammation in RAW264.7 cells and mouse models. Int Immunopharmacol. 2015; 26(2):286-94.

8. Verma N, Verma R, Kumari R, Ranjha R, Paul J. Effect of salicin on gut inflammation and on selected groups of gut microbiota in dextran sodium sulfate-induced mouse model of colitis. Inflamm Res. 2014; 63(2):161-9.

9. $\mathrm{Hu}$ R, Hilakivi-Clarke L, Clarke R. Molecular mechanisms of tamoxifen-associated endometrial cancer (Review). Oncol Lett. 2015; 9(4):1495-1501.

10. Costa R, Costa-Filho RB, Talamantes SM, et al. Interstitial Pneumonitis Secondary to Trastuzumab: A Case Report and Literature Review. Case Rep Oncol. 2017;10(2):524-30.

11. Peter Schmid, Sylvia Adams, Hope S. Rugo, Andreas Schneeweiss, Carlos H. Barrios, Hiroji Iwata, Veronique Dieras, Volkmar Henschel, Luciana Molinero, Stephen Y. Chui, Amreen Husain, Eric P. Winer, Sherene Loi, Leisha A. Emens. IMpassion130: updated overall survival (OS) from a global, randomized, double-blind, placebo-controlled, Phase III study of atezolizumab (atezo) + nab-paclitaxel (nP) in previously untreated locally advanced or metastatic triple-negative breast cancer (mTNBC). Journal of Clinical Oncology. 2019; 37(15): suppl. 1003. 
12. Liu B, Ezeogu L, Zellmer L, Yu B, Xu N, Joshua Liao D. Protecting the normal in order to better kill the cancer. Cancer Med. 2015;4(9):1394-1403.

13. Sinha S, Sharma S, Vora J, Shah H, Srivastava A, Shrivastava N. Mucuna pruriens (L.) DC chemo sensitize human breast cancer cells via downregulation of prolactin-mediated JAK2/ STAT5A signaling. J Ethnopharmacol. 2018; 217:23-35.

14. Carballo JL, Hernández-Inda ZL, Pérez P, GarcíaGrávalos MD. A comparison between two brine shrimp assays to detect in vitro cytotoxicity in marine natural products. BMC Biotechnol. 2002; 2:17.

15. Onwuamah C, Ekama S, Audu R, Ezechi O, Poirier M, Odeigah P. Exposure of Allium cepa Root Cells to Zidovudine or Nevirapine Induces Cytogenotoxic Changes. PLoS ONE. 2014;9(3): e90296.

16. Horwitz KB, Costlow ME, McGuire WL. MCF-7; a human breast cancer cell line with estrogen, androgen, progesterone, and glucocorticoid receptors. Steroids. 1975; 26(6):785-95.

17. Eamon J. Mahdi, Abdulrahman M. Alshahrani, Asma A. Abdulsatar, Jassem G. Mahdi, Morphological evaluation of apoptosis induced by salicylates in HT-1080 human fibrosarcoma cells, Journal of Microscopy and Ultrastructure. 2014; 2 (1):20-27.

18. Wong RS. Apoptosis in cancer: from pathogenesis to treatment. J. Exp Clin Cancer Res. 2011;30(1):87.

19. Elmore S. Apoptosis: a review of programmed cell death. Toxicol Pathol. 2007;35(4):495-516.

20. Sabaa M, ELFayoumi HM, Elshazly S, Youns M, Barakat W. Anticancer activity of salicin and fenofibrate. Naunyn Schmiedebergs Arch Pharmacol. 2017;390(10):1061-71.

21. Hostanska K, Jürgenliemk G, Abel G, Nahrstedt A, Saller R. Willow bark extract (BNO1455) and its fractions suppress growth and induce apoptosis in human colon and lung cancer cells. Cancer Detect Prev. 2007;31(2):129-39.

22. J.G. Mahdi, N.M. A 1Musayeib, E.J. Mahdi, C.J. Pepper. Pharmacological importance of simple phenolic compounds on inflammation, cell proliferation and apoptosis with a special reference to $\beta$-d-Salicin and hydroxybenzoic acid. European Journal of Inflammation. 2013; 11(2):327-36.

23. Karin M. NF-kappaB as a critical link between inflammation and cancer. Cold Spring Harb Perspect Biol. 2009;1(5): a000141.

24. Bonaterra GA, Kelber O, Weiser D, Metz J, Kinscherf R. In vitro anti-proliferative effects of the willow bark extract S T W 33 - I . Arzneimittelforschung. 2010;60(6):330-5.

25. Baud V, Karin M. Is NF-kappaB a good target for cancer therapy? Hopes and pitfalls. Nat Rev Drug Discov. 2009;8(1):33-40.

26. Meyer BN, Ferrigni NR, Putnam JE, Jacobsen LB, Nichols DE, McLaughlin JL. Brine shrimp: a convenient general bioassay for active plant constituents. Planta Med. 1982; 45(5):31-4.

27. Enayat S, Ceyhan MŞ, Başaran AA, Gürsel M, Banerjee S. Anticarcinogenic effects of the ethanolic extract of Salix aegyptiaca in colon cancer cells: involvement of Akt/PKB and MAPK pathways. Nutr Cancer. 2013;65(7):1045-58.

28. Welsh J. Animal Models for Studying Prevention and Treatment of Breast Cancer, Animal Models for the Study of Human Disease. 1 ${ }^{\text {st }}$ ed. Academic Press, 2013. 997-1018p.

29. Kimura, M., Yoshioka, T., Saio, M. Y Banno, H Nagaoka \& Y Okano et al. Mitotic catastrophe and cell death induced by depletion of centrosomal proteins. Cell Death Dis 4, e603 (2013).

30. Fragkos M, Beard P. Mitotic Catastrophe Occurs in the Absence of Apoptosis in p53-Null Cells with a Defective G1 Checkpoint. PLoS ONE. 2011,6(8);e22946.

31. Maiti, S., Nazmeen, A. Impaired redox regulation of estrogen metabolizing proteins is important determinant of human breast cancers. Cancer Cell Int 19, 111 (2019).

32. Nazmeen, A., S. Maiti, S. Synergism between BRCA1 Mutation and Impaired Estrogen Signaling in Oxidative Stress Modulation Makes Estrogen Responsive Tissues (Breast) More Susceptible to Develop Cancer. Journal of Cell Science \& Therapy. 2018. 09(04).

33. Nazmeen A, Maiti S. Oxidant stress induction and signalling in xenografted (human breast cancertissues) plus estradiol treated or N-ethyl-Nnitrosourea treated female rats via altered estrogen sulfotransferase (rSULT1E1) expressions and SOD1/catalase regulations. Mol Biol Rep. 2018;45(6):2571-84.

34. Nazmeen A., Chen G., Ghosh T.K, Maitri S. Breast cancer pathogenesis is linked to the intra-tumoral estrogen sulfotransferase (hSULT1E1) expressions regulated by cellular redox dependent $\mathrm{Nrf}-2 / \mathrm{NF} \kappa \beta$ interplay. Cancer Cell International 20. 2020; 70 (2020).

35. El-Shemy HA, Aboul-Enein AM, Aboul-Enein KM, Fujita K (2007) Willow Leaves' Extracts Contain Anti-Tumor Agents Effective against Three Cell Types. PLOS ONE 2(1): e178.

36. Applanat MP, Buteau-Lozano H, Herve MA, Corpet A. Vascular endothelial growth factor is a target gene for estrogen receptor and contributes to breast cancer progression. Adv Exp Med Biol. 2008;617:437-44.

37. Barontini J, Antinucci M, Tofanelli S, Cammalleri M, Monte M, Gemignani F, Vodicka P, Marangoni R, Vodickova L, Kupcinskas J, Vymetalkova V, Forsti A, Canzian F, Stein A, Moreno V, Mastrodonato N, Tavano F, Panza A, Barale R, Landi S \& Campa D. Association between polymorphisms of TAS2R16 and susceptibility to colorectal cancer. BMC Gastroenterol.17, 2017;104.

38. Kong CS, Kim KH, Choi JS, Kim JE, Park C, Jeong JW. Salicin, an extract from white willow bark, 
inhibits angiogenesis by blocking the ROS-ERK pathways. Phytother Res. 2014;28(8):1246-51.

39. Bounaama A, Enayat S, Ceyhan MS, Moulahoum $\mathrm{H}$, Djerdjouri B, Banerjee S. Ethanolic Extract of Bark from Salix aegyptiaca Ameliorates 1,2dimethylhydrazine-induced Colon Carcinogenesis in Mice by Reducing Oxidative Stress. Nutr Cancer. 2016;68(3):495-506.

40. Ke-feng Zhai, Hong Duan, Ghulam Jilany Khan, Hui Xu, Fang-kai Han, Wen-gen Cao, Gui-zhen Gao, Ling-ling Shan, and Zhao-Jun Wei Journal of Agricultural and Food Chemistry. 2018;66 (24), 6073-82.

41. Muthuswamy SK, Li D, Lelievre S, Bissell MJ, Brugge JS. ERBB2, but not ErbB1, reinitiates proliferation and induces luminal repopulation in epithelial acini. Nat Cell Biol. 2001;3(9):785-92.

42. Woods Ignatoski KM, Grewal NK, Markwart S, Livant DL, Ethier SP. p38MAPK induces cell surface alpha4 integrin downregulation to facilitate erbB-2-mediated invasion. Neoplasia (New York, N.Y.). 2003;5(2):128-34.

43. Moasser, M. The oncogene HER2: its signaling and transforming functions and its role in human cancer pathogenesis. Oncogene 26, 2007; 6469-87.

44. Graham JD, Yeates C, Balleine RL, Harvey SS, Milliken JS, Bilous AM, Clarke CL. Characterization of progesterone receptor $\mathrm{A}$ and $\mathrm{B}$ expression in human breast cancer. Cancer Res. 1995;55(21):5063-8.

45. Jacobsen BM, Richer JK, Sartorius CA, Horwitz KB. Expression profiling of human breast cancers and gene regulation by progesterone receptors. J Mammary Gland Biol Neoplasia. 2003;8(3):257-68.

46. Jacobsen BM, Richer JK, Schittone SA, Horwitz KB. New human breast cancer cells to study progesterone receptor isoform ratio effects and ligand-independent gene regulation. J Biol Chem. 2002;277(31):27793-800.
47. Brisken C. Progesterone signalling in breast cancer: a neglected hormone coming into the limelight. Nat Rev Cancer. 2013;13(6):385-96.

48. Nolan E, Vaillant F, Branstetter D, Pal B, Giner G, Whitehead L, Lok SW, Mann GB; Kathleen Cuningham Foundation Consortium for Research into Familial Breast Cancer (kConFab), Rohrbach K, Huang LY, Soriano R, Smyth GK, Dougall WC, Visvader JE, Lindeman GJ. RANK ligand as a potential target for breast cancer prevention in BRCA 1-mutation carriers. Nat Med. 2016;22(8):933-9.

49. Sau A, Lau R, Cabrita MA, Nolan E, Crooks PA, Visvader JE, Pratt MA. Persistent Activation of NF$\kappa \mathrm{B}$ in BRCA1-Deficient Mammary Progenitors Drives Aberrant Proliferation and Accumulation of DNA Damage. Cell Stem Cell. 2016;19(1):52-65.

50. Tanos T, Sflomos G, Echeverria PC, Ayyanan A, Gutierrez M, Delaloye JF, Raffoul W, Fiche M, Dougall W, Schneider P, Yalcin-Ozuysal O, Brisken C. Progesterone/RANKL is a major regulatory axis in the human breast. Sci Transl Med. 2013;5(182):182ra55.

51. Asselin-Labat ML, Vaillant F, Sheridan JM, Pal B, Wu D, Simpson ER, Yasuda H, Smyth GK, Martin TJ, Lindeman GJ, Visvader JE. Control of mammary stem cell function by steroid hormone signalling. Nature. 2010;465(7299):798-802.

52. Groshong SD, Owen GI, Grimison B, Schauer IE, Todd MC, Langan TA, Sclafani RA, Lange CA, Horwitz KB. Biphasic regulation of breast cancer cell growth by progesterone: role of the cyclindependent kinase inhibitors, p21 and p27(Kip1). Mol Endocrinol. 1997;11(11):1593-607.

53. Hissom JR, Moore MR. Progestin effects on growth in the human breast cancer cell line T-47D--possible therapeutic implications. Biochem Biophys Res Commun. 1987;145(2):706-11. 